\title{
UCF Smart Mailbox: Reinforcing Communications in the Neighborhoods
}

\author{
Anastasia Angelopoulou ${ }^{1}$, Konstantinos Mykoniatis ${ }^{1}$, Karen Carlson ${ }^{2}$, \\ and Si-Jung Kim² \\ ${ }^{1}$ Modeling \& Simulation Graduate Program, University of Central Florida, \\ Orlando, FL 32816, USA \\ \{kmykoniatis, aangelopoulou\}@knights.ucf .edu \\ ${ }^{2}$ Digital Media - School of Visual Arts and Design, University of Central Florida, \\ Orlando, FL 32816, USA \\ karenjcarlson@gmail.com, sjkim@ucf.edu
}

\begin{abstract}
Commonplace objects are being redesigned with digital functionality. Near invisible networks of radio frequency identification tags (RFID) are being deployed on almost every type of consumer product, and the "Ambient intelligence" promises to form a global network of physical objects as ubiquitous as the worldwide web itself. This diverse global network, "the internet of things" (IoT), provides digital connectivity on top of existing infrastructure and items. Nascent "smart object" developments like the presented "UCF Smart Mailbox," not only reformulate our relationship with the objects themselves, but they can also support social relationships that contribute to the sustainability of the society.
\end{abstract}

Keywords: Arduino, mailbox, sensors, audio, physical interaction, neighborhood, communications, temperature, notifications.

\section{Introduction}

In February 2013, the United States Post Office made a historic announcement: Saturday delivery will cease by August [1]. This follows a downward trend in mail so sharp that some speculate that paper mail be obsolete in the future. We look to the mailbox: the symbol of residential communication for two centuries, and ask a simple question: how can a mailbox be used to support communication between neighbors and strengthen community?

The answer lies in new media technology, the very technology often blamed for the breakdown of community in the 21 st century neighborhood. "As the Internet further reduces the burden of distance, it may further degrade the role of the parochial realm; ties across the street may become increasingly rare as ties at a distance become ever more accessible" [2]. Digital technologies, while uniting people across distances, can also be used to reinforce place-based communities.

Urban planning and digital media practitioners have joined forces in the past several years to recast public spaces with tools and technologies to promote 
information sharing, community pride and play [3]. Examples of experimentation abound. Consider Mouna Andraos and Melissa Mongiat's "21 Swings", an urban installation in Montreal, Canada, where swinging generates a melody, but one that is best experienced communally, when many swings are engaged [4]. Imagine how many dreary bus stops could be improved with a musical swing installation: where people aren't shutting out the world by listening to music from their ipods and earbuds, but making music together, by moving their bodies.

Another whimsical application of digitally augmented public space is the Piano Staircase in Berlin, Germany, by Volkswagon. The stairs are painted like a piano and music sounds as they are stepped on. The designers of this installation had more than music in mind. They wanted to create an incentive for people to use the stairs, instead of the escalator, by making the stairs more fun. They were able to increase stair usage by $66 \%[5]$.

While the bulk of urban digital projects have focused on public places, some have concentrated on the domain of the neighborhood. Digital devices join a long line of technology blamed for the breakdown of community, including the air conditioner and television. Now smart phones and social media fulfill our communication needs without real time interaction or geographic proximity.

Can digital experiences draw people back outside and into communication patterns with their neighbors? This is the goal of The Smart Mailbox. Can the mailbox become its owners face and voice in a faceless community? Apostol et al. in "From FaceBlock to Facebook or the Other Way Around," provide more fodder for our discussion: "We wish to employ the technology developed for such online communities to bring communities back to the "barn-raising"-type of collective action that could encourage participation, increase the feelings of solidarity and social capital, and lead to building community identity" [6]. As electronic mailboxes continue to innovate at rapid pace, physical mailboxes have changed little in the last century. Sensors implanted into everyday objects are changing the way we interact with our homes, transforming them into smart communicators [7]. Why should the mailbox be left behind?

We are not the first to ask this question. Denzil Ferraira, a computer science student at the University of Oulu in Finland created SmartMail: a digital physical mail. Using an ArduinoDuemilanove and a photosensitive sensor, he developed an electronic notification reporting "you've got mail" for physical mail [8].

\section{Prototype Design Description and Implementation}

This section describes the design process of the UCF Smart Mailbox, including the implemented algorithms, the hardware architecture and the prototype implementation. The UCF Smart Mailbox system is able to play a Personal Message that the owner wants to share with the community and also to record a message from the neighbors. If a message is recorded, the system notifies the owner for the recorded message. More specifically, a Twitter notification is sent to the owner via email. Twitter notifications about the outdoor environment's temperature and receiving a new mail are sent as well. 
Software. Several algorithms were developed, such as for playing an audio file and recording a message. The mailbox plays an audio message if a person is detected near the mailbox (distance less than $1 \mathrm{~m}$ ). Another algorithm waits for input in order to allow the message recording. While the user presses the recording button, a message is being recorded. An LED turns on to indicate that a recording takes place. When the button is released, a Twitter notification is sent to the owner and the LED turns off to indicate that the recording is over. Algorithms for providing information about the temperature and the changes in light conditions were also implemented. The overall behavior of the UCF Smart Mailbox system is described using a UML diagram. The UML use case diagram (Fig. 1) illustrates the events that occur in order for the UCF Smart Mailbox owner to leave and/or receive messages from the neighbors.

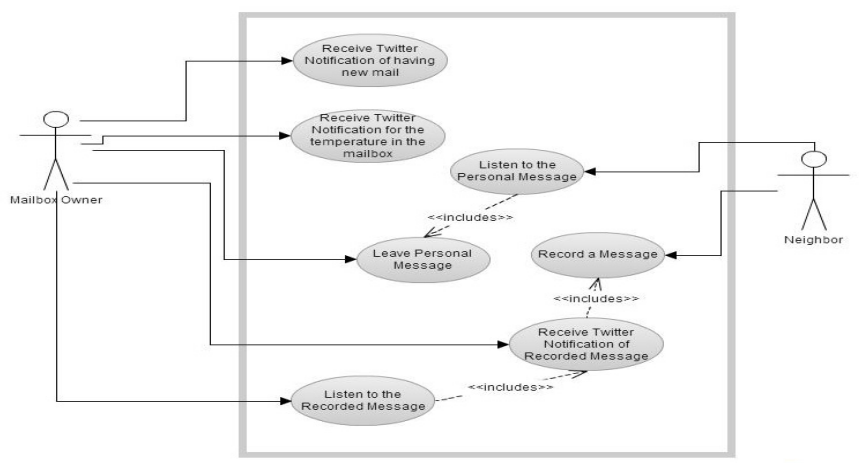

Fig. 1. UML Diagram for the UCF Smart Mailbox System

Hardware Architecture. The configuration of the hardware consists of a Rugged audio shield mounted on the Arduino board. A speaker and a microphone are connected on the Rugged Audio Shield for playing and recording a message, respectively. An XBee shield is placed on top of the Rugged Audio Shield. A proximity sensor and a force sensitive sensor are connected to the Xbee shield as inputs. Two XBees were used to send and receive signals for all the Twitter notifications. An LED is used to indicate that the message is recorded. A temperature sensor and a photocell provide information about the temperature and the changes in light conditions. The architecture diagram (Fig. 2) illustrates an internal view of the system architecture in order to understand the different components of the UCF Smart Mailbox's components and how they interact.

Prototype. The UCF Smart Mailbox prototype has been developed to give neighbors a geography-based tool for communication and interaction. The Mailbox exhibits the following functionalities:

- Proximity-triggered message broadcast

- Push button-triggered recording device for leaving a message

- Notification to owner via digital social network Twitter that a message has been delivered

- Notification to owner via digital social network Twitter that physical mail has been delivered

- Notification to owner of temperature at mailbox 


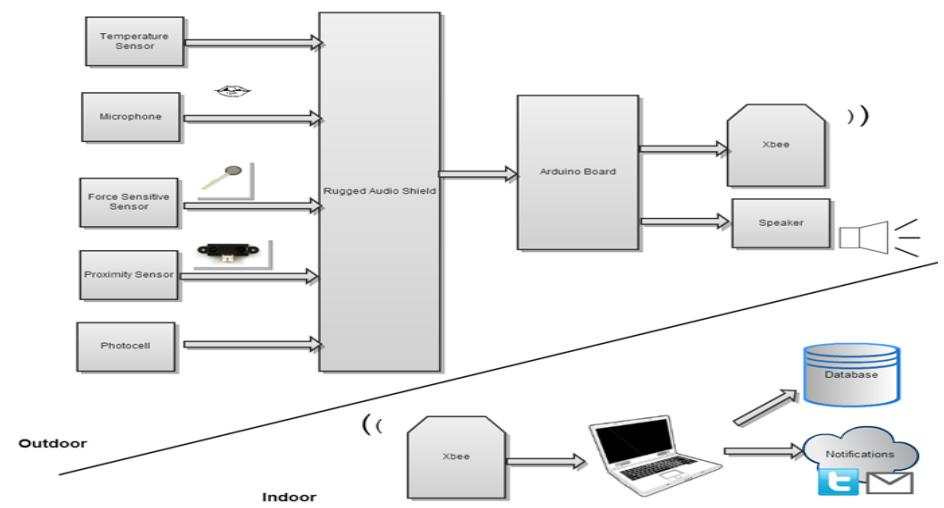

Fig. 2. UCF Smart Mailbox Architecture

Fig. 3 illustrates the different components and the implemented prototype. Except from the visual components, the prototype includes a Twitter-python script for sending the Twitter notifications and storing information in a database for future data manipulation.
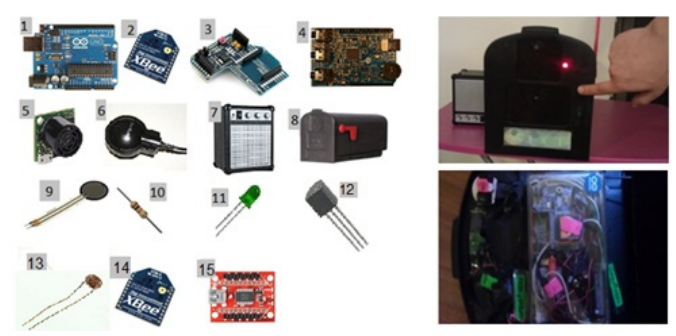

Fig. 3. UCF Smart Mailbox Components \& Prototype Representation

\section{$3 \quad$ Pilot Testing and Results}

The Smart Mailbox prototype was initially tested with 12 Central Florida community members. The participant population was all between the ages of 18-35 and included an equal number of males and females. Quantitative and qualitative data was collected in the form surveys. The experimenters also took notes during user-mailbox interaction to document usability issues. The experimental process consisted of three steps:

- Step One (Baseline Survey): Participants were asked to fill out a pre-demo survey about their residential status and communication patterns with neighbors.

- Step Two (Exposure to Mailbox): Participants were given verbal instructions from the experimenters about the Smart Mailbox. They were instructed to approach the mailbox, listen to the broadcast and leave a message for the mailbox owner. 
- Step Three (After Exposure): After using the Smart Mailbox, participants were asked to complete the second survey. This survey assessed usability, feature set preference on the part of users and user projection about how they believed the Smart Mailbox may influence their neighborhood communication patterns.

After the collection and the cleaning of the raw data, we applied basic statistics to analyze the data. More specifically, the analysis included descriptive statistics such as mean, median, mode, and min/max, computing means (averages) and standard deviations, computing proportions and percentages, and drawing histograms or pie charts.

Mailbox Impact on Meeting New People. Users predict the Smart Mailbox will improve the likelihood of meeting new people in the next month. We compared the likelihood of meeting new people with and without using the Smart Mailbox, resulting in an average rating of 4.36 vs. 2.80 on a scale of 1-5. Males were more likely to rate the Smart Mailbox's potential to improve relationships, than females. Females averaged 3 (a few times a month), males averaged 4 (a few times a week). Users also predict they will walk around the neighborhood more if it had Smart Mailboxes (Fig. 4).
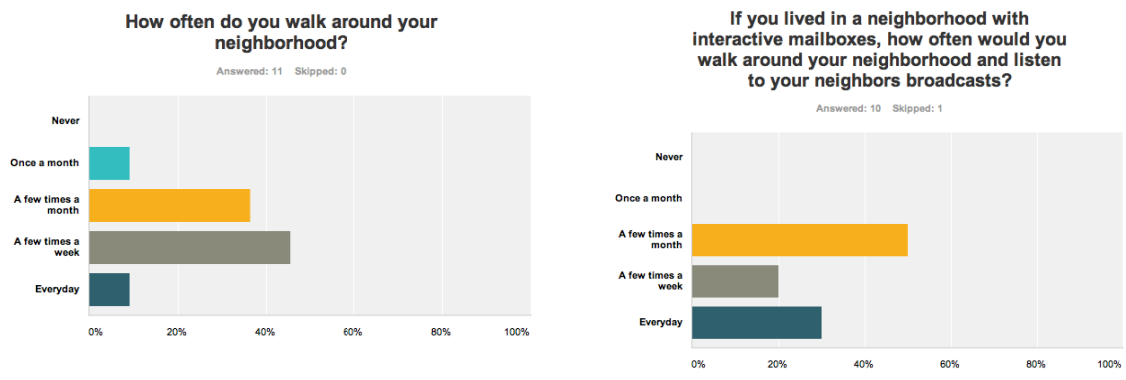

Fig. 4. Results about likelihood of walking around the neighborhood more

Feature Popularity. Ability to leave a message, hear a message and be notified when you receive mail all received equal average rating of 2.2 on a scale of 1-5 (1 being most useful, 5 being least useful). Ability to receive a temperature reading was the least popular feature and received a rating of 3.4.

Usability. While gender was not a focus of our study, we did collect demographic information that included gender. Males and Females rated most questions the same, however males found the Smart Mailbox significantly easier to use than females. On a scale of 1-5 (5=easy, 1=Difficult) males averaged 4.333 and females averaged 2.666. It would be interesting to see if this is repeated with a larger sample size.

Sociability Correlation to Type of Housing. We predicted that the type of housing a user lived in may influence their baseline level of sociability as well as their predictions about how the Smart Mailbox would influence their sociability. We did not have a large sample size, but can see from pilot data that single family home 
dwellers are more likely to know their neighbors and communicate with them than dorm and apartment dwellers. This may be explained by the temporary status of apartment and dorm living vs. the more permanent or 'settled' nature of single-family home dwellers. We did not ask whether single-family home dwellers owned their homes. That may be a question for a future survey. Perhaps owners are more likely to invest time and energy in getting to know their neighbors.

\section{Conclusions and Future Work}

A larger phase experiment could be conducted in the future to see if networked interactive mailboxes improve a community's sense of cohesiveness. We could track inter-community interactions and relationships before and after the installation to understand how the mailbox facilitates neighborliness. In the future, the UCF Smart mailbox system will integrate an identification and tracking module that will allow interaction with the users only if they carry an RFID tag. Future work may include transforming the recorded WAV messages to byte array, transmitting them to the receiver XBee, converting the byte array to WAV file and saving them in the database in order for the user to listen to them on a computer or a portable device. Future plans may also include the implementation of a blog/website to display and analyze the interactions that occur in the community. Data from different neighborhoods in the world could be displayed and compared. Moreover, we could identify and display which neighborhoods have "stronger" interactions. The content and number of "Personal Messages" as well as the number of recorded messages could be used for evaluating the activity network and drawing conclusions about the behavior of the neighborhood mailbox network.

\section{References}

1. Nixon, R.: Postal Service Plans to End Saturday Delivery. New York Times (2013), http: / / www . nytimes. com/2013/02/07/us/postal-service-plansto-end-saturday-delivery.html?_r=0 (retrieved on February 27, 2013)

2. Hampton, K.: Neighborhoods in the Network Society the e-Neighbors study. Information, Communication \& Society 10(5), 714-748 (2007)

3. Klaebe, H.G., Adkins, B.A., Foth, M., Hearn, G.N.: Embedding an ecology notion in the social production of urban space. In: Handbook of Research on Urban Informatics: The Practice and Promise of the Real-time City, pp. 179-194 (2009)

4. Andraos, M., Mongiat, M.: 21 Balançoires (2012), http://www. dailytouslesjours .com/project/21-balancoires / (retrieved on February 27, 2013)

5. Volkswagon: Piano Staircase. The Fun Theory (2009), http: / / www . thefuntheory . $\mathrm{com} /$ (retrieved on February 20, 2013)

6. Apostol, I., Antoniadis, P., Banerjee, T.: From Face-Block to Facebook or the other Way Around? In: Proceedings of Sustainable City and Creativity, Promoting Creative Urban Initiatives, Naples (2008)

7. Chan, M., Estève, D., Escriba, C., Campo, E.: A review of smart homes-Present state and future challenges. Comp. Methods and Programs in Biomedicine 91(1), 55-81 (2008)

8. Ferreira, D.: SmartMail-digital physical mail, http://www. denzilferreira.com/ smart-mail/ (retrieved on February 22, 2013) 